\title{
Applications of Few-Hundred Microarcsecond VLBI Astrometry: Planetary Relativistic Deflection, PPN Gamma Determination and Deep-Space Tracking
}

\author{
S. T. Lowe and R. N. Treuhaft \\ Radio Metric Tracking Applications Section \\ Jet Propulsion Laboratory, 238-700 \\ California Institute of Technology \\ Pasadena, California 91109
}

This paper presents several applications of a few hundred microarcsecond ( $\mu$ as) astrometric technique which has been developed and demonstrated using differential very long baseline interferometry (VLBI). A brief description of the technique along with several applications will be discussed below. This technique was developed for high-accuracy deep-space tracking ${ }^{[1]}$, but the first application tracked an extragalactic radio source in a measurement of Jovian relativistic deflection ${ }^{[2]}$. Current work includes making a state-of-the-art solar deflection measurement, and thus, an improved determination of the Parameterized Post Newtonian (PPN) gamma parameter ${ }^{[3]}$. A number a future spacecraft tracking applications, described below, are also enabled by this technique.

The primary observable measured with VLBI is the difference in arrival times of a radio wave between two radio telescopes. The baseline vector between the two telescopes, $\vec{B}$, has a length on the order of $10000 \mathrm{~km}$. If $\hat{s}$ is a unit vector pointing to the radio source, then the measured time delay, $\tau$, can be written

$$
\tau=\frac{-1}{c} \vec{B} \cdot \hat{s}
$$

where $c$ is the speed of light. From this equation, one can see that delay measurements can yield information on $\vec{B}$, giving geodetic results, and on $\hat{s}$, resulting in astrometric measurements.

The few-hundred $\mu$ as astrometric technique presented here depends on defining a local inertial reference frame, defined by several natural extragalactic radio sources. These sources are located throughout the observing station's mutual visibility region, and represents about a $30^{\circ} \times 30^{\circ}$ field in the sky. The "target" source, which can be a natural radio source or a spacecraft, is defined as the radio source whose position one wishes to measure relative to the local frame. As described below, measurements of the target's position are made at two different epochs and differenced so the position shift of the target is the final measured quantity. 
To make astrometric measurements at the few hundred $\mu$ as level requires aggressively eliminating dominant systematic errors. The best a priori models of the delay measurements are used, followed by a least squares estimation for parameters associated with known physical effects, such as station clock drifts, earth orientation and tropospheric delays, which effectively improve the model. By making two identical measurements, in the sidereal sense, and calculating the position shift of the target, many constant, unmodeled errors cancel. The most sensitive instrumentation, the Deep Space Network (DSN) antennas (70 $\mathrm{m}, 20^{\circ} \mathrm{K}$ ), are also used to reduce white system noise. The two dominant error sources remaining are index-of-refraction fluctuations in the wet troposphere, and system noise. Both of these error sources can be modeled a priori, so the measurement error covariance is not artificially inflated, as is done with most VLBI analyses. Finally, the contribution to the target shift can be estimated by using a sensitivity analysis. This allows one to determine how well unmodeled errors, such as station location or antenna deformation errors, cancel when forming the differential positions.

The first application of this technique was planetary (Jovian) relativistic deflection. Because this work was published last year ${ }^{[1]}$, only a quick review will be given here. The physics behind this measurement, from the General Relativity point of view, is that Jupiter's mass gives rise to a local space-time curvature. This curvature causes electromagnetic radiation passing near the planet to experience a small angular deflection. On 21 March 1988, Jupiter passed within 200 arcseconds ( $\approx 10$ Jovian radii) of radio source P $0210+113$. Such an occultation occurs about twice per decade, on average. Ten measurements of the target position were made on that date over the 4 hours of mutual visibility. The average deflection, projected onto the California-Australia baseline used here, was about $300 \mu$ as. About two weeks later, on 2 April 1988, the observation sequence was repeated. On this date, Jupiter appeared about $1^{\circ}$ from the target, and the average deflection was about $20 \mu$ as. Each of the 10 measurements had an average accuracy of 160 $\mu$ as. Tropospheric fluctuations and white system noise contributed about $145 \mu$ as and 70 $\mu$ as to this error, respectively. The results are shown in Figure 1. The reduced $\chi^{2}$ is 0.6 for the General Relativity hypothesis and 4.1 for the no deflection hypothesis. This rejects the no deflection hypothesis with $99.999 \%$ confidence.

Efforts to apply this technique to an improved solar deflection measurement are currently underway. If 10 measurements with $160 \mu$ as accuracy can be made $3^{\circ}$ from the sun, where solar deflection is about 150 milliarcseconds (mas), then the potential exists to improve the $0.1 \%$ solar deflection reported by the best measurement to date ${ }^{[4]}$. It should be noted that the referenced $0.1 \%$ solar deflection measurement used more than 300000 VLBI observations taken over a ten year period; the Jovian experiment required about 80 observations taken over 8 hours. The main technical problem to over come for observations near the sun is that solar plasma fluctuations cause large phase excursions for time scales on the order of .1 seconds at X-band and 0.02 seconds at S-band ${ }^{[5]}$. This problem has been partially solved by processing the data using a modification to the JPL/CIT Block II 
correlator; one can now obtain cross-correlation phasors every 0.025 seconds. By looking at a strong source with DSN antennas, significant phase determinations can be made in a few milliseconds. Test data has been taken by observing a bright source $3^{\circ}$ from the sun and has verified that, at X-band, phase tracking is easily accomplished using 0.025 second integrations. However, S-band still only shows partial phase coherence at these time scales. If $\mathrm{S}$ and X-band group delays are calculated and used to calibrate charged particle effects, the RMS delay scatter is about 12 times higher than that seen for a source far from the sun. The standard dual frequency (S and X-band) calibration of dispersive plasma effects, which assumes a constant total electron content (TEC) over the scan duration, was found not to be an adequate model. The next order term, proportional to the TEC rate, was found to be significant for data near the sun but not for observations far from the sun. Correcting the data with this additional term significantly reduced the delay RMS in all cases tried. Using this and other analysis techniques to improve the TEC modeling resulted in a reduction in the RMS scatter of the scans $3^{\circ}$ from the sun. The scatter was still 4 times the expected scatter for sources far from the sun, however. The possibility of getting the Block II correlator to produce phasors every 0.005 seconds being explored now. This may significantly improve the S-band delays, and thus further reduce the delay RMS scatter. For the future, plans to make a full measurement of solar deflection using this technique are being made to determine the limiting error sources and potential deflection sensitivity. Some possible experimental parameters to be explored are the optimal sun-earth-source angel and solar latitudes used for such measurements. Perhaps moving to $4^{\circ}$ from the sun would significantly improve the S-band phase coherence without sacrificing the deflection signal too much, or phase coherence may improve by observing sources at higher solar latitudes.

This few-hundred $\mu$ as technique opens up many potential deep-space tracking applications for missions to the outer planets (for reference, a $160 \mu$ as angular error corresponds to a 580 meter transverse position error at Jupiter). Current operational spacecraft tracking has angular errors on the order of 10 mas; a factor of 50 improvement could be possible if this technique were used. One problem in tracking spacecraft to the outer planets is that the spacecraft position is known in the radio reference frame while the planet's position is known in the optical frame. This technique could be used to better determine the phase offsets between the two frames by observing a spacecraft in orbit or on the surface of a planet. Similarly, a number of position measurements of a spacecraft as it approaches a planet could be used to determine the planet's position in the radio frame through the signature of gravity acting on the spacecraft. Some missions plan to release probes as they approach a planet. Position measurements of the spacecraft before and after the probe release can be used to reconstruct the probe's trajectory. Such a measurement is planned for Galileo's probe release as it approaches Jupiter in December 1995, but this will require the on-board high-gain antenna which has failed to deploy so far. The Cassini mission, which is to study Saturn and its rings and satellites could use this technique to better measure the ring radii, which is a limiting error source in some ring dynamics 
models. Finally, missions could greatly reduce on-board fuel (and thus weight) if they could enter orbit by using a planet's atmospheric drag to slow it down, rather than by firing thrusters. Aerocapture, as this is called, would require high-accuracy navigation to guide the spacecraft into a narrow window in the upper atmosphere.

In summary, a radio metric technique with $160 \mu$ as accuracy has been developed and demonstrated. This technique was used to make the first measurement of planetary relativistic deflection. The dominant error sources were modeled, so no arbitrary error inflation was necessary. Work is now in progress to make an improved solar deflection measurement which could improve the determination of PPN gamma. This technique has also enabled several spacecraft tracking applications. Work is also underway to reduce the two accuracy-limiting error sources; a large effort has begun to build an improved water vapor radiometer (WVR) which could be used to measure and calibrate water vapor effects in the VLBI observables, and work is in progress to study intercontinental phase connection so the much more accurate phase delay observable could be used in place of the group delay.

\section{References}

1. Treuhaft, R. N., "Deep Space Tracking in Local Reference Frames," Jet Propulsion Laboratory TDA Progress Report 42-94, p. 1-15, 1988.

2. Treuhaft, R. N. and Lowe, S. T., "A Measurement of Planetary Relativistic Deflection," Astronomical Journal, 105, p. 1879, 1991.

3. Will, C. M., "Theoretical Frameworks for Testing Relativistic Gravity, II. Parameterized Post-Newtonian Hydrodynamics, and the Nordtvedt Effect," Astrophysical Journal, 163, p. 611, 1971.

4. Robertson, D. S., Carter, W. E. and Dillinger, W. H., "New Measurement of Solar Gravitational Deflection of Radio Signals Using VLBI," Letters to Nature, 349, p. $768,1991$.

5. Woo, R. and Armstrong, J. W., "Spacecraft Radio Scattering Observations of the Power Spectrum of Electron Density Fluctuations in the Solar Wind," Journal of Geophysical Research, 84, p. 7288-7296, December 1979. 


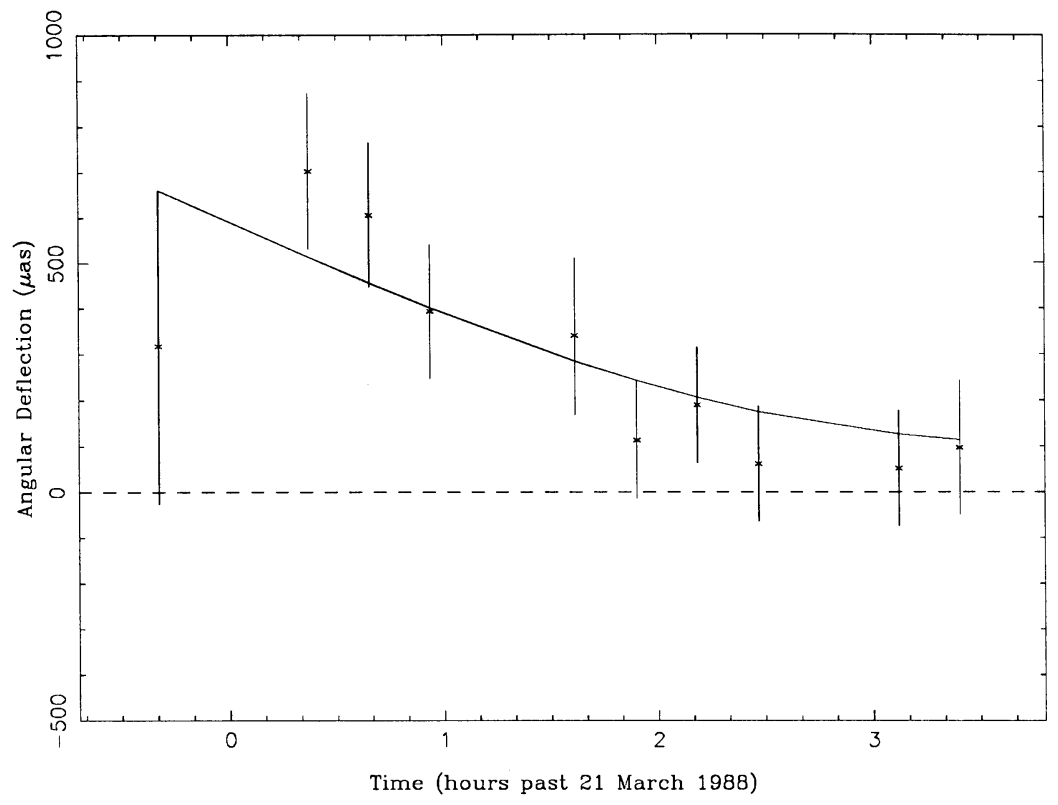

Fig. 1 Results from the Jovian relativistic deflection measurement. The points with error bars are the 10 measurements, the curved solid line shows the prediction from General Relativity, and the dashed line shows the no-deflection hypothesis. 